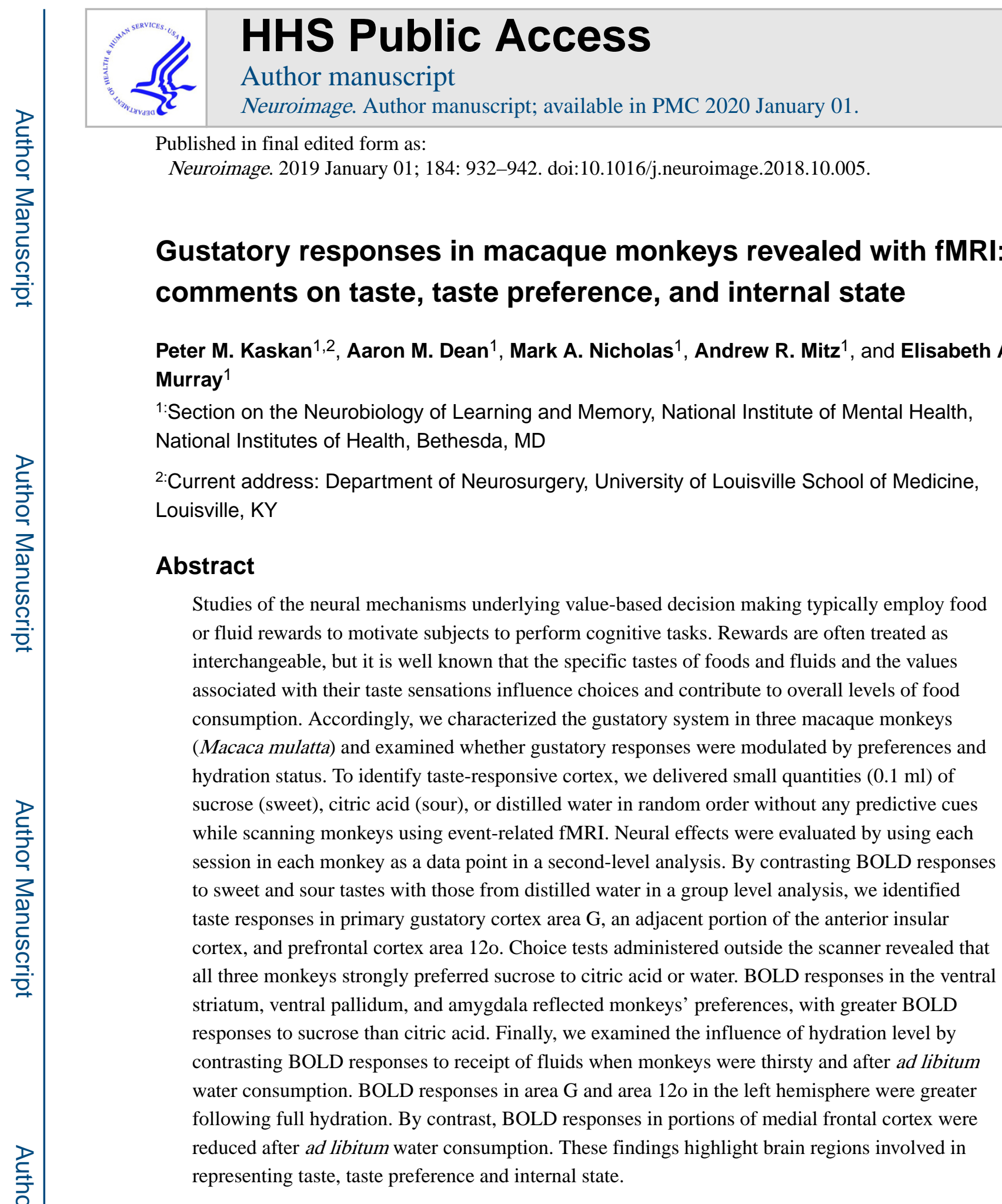

Published in final edited form as:

Neuroimage. 2019 January 01; 184: 932-942. doi:10.1016/j.neuroimage.2018.10.005.

\title{
Gustatory responses in macaque monkeys revealed with fMRI: comments on taste, taste preference, and internal state
}

\author{
Peter M. Kaskan ${ }^{1,2}$, Aaron M. Dean ${ }^{1}$, Mark A. Nicholas ${ }^{1}$, Andrew R. Mitz ${ }^{1}$, and Elisabeth A. \\ Murray \\ Section on the Neurobiology of Learning and Memory, National Institute of Mental Health, \\ 2:Current address: Department of Neurosurgery, University of Louisville School of Medicine, \\ Louisville, $\mathrm{KY}$
}

\section{Abstract}

Studies of the neural mechanisms underlying value-based decision making typically employ food or fluid rewards to motivate subjects to perform cognitive tasks. Rewards are often treated as interchangeable, but it is well known that the specific tastes of foods and fluids and the values associated with their taste sensations influence choices and contribute to overall levels of food Macaca mulatta) and examined whether gustatory responses were modulated by preferences and hydration status. To identify taste-responsive cortex, we delivered small quantities $(0.1 \mathrm{ml})$ of sucrose (sweet), citric acid (sour), or distilled water in random order without any predictive cues while scanning monkeys using event-related fMRI. Neural effects were evaluated by using each session in each monkey as a data point in a second-level analysis. By contrasting BOLD responses to sweet and sour tastes with those from distilled water in a group level analysis, we identified taste responses in primary gustatory cortex area $\mathrm{G}$, an adjacent portion of the anterior insular cortex, and prefrontal cortex area 12o. Choice tests administered outside the scanner revealed that all three monkeys strongly preferred sucrose to citric acid or water. BOLD responses in the ventral striatum, ventral pallidum, and amygdala reflected monkeys' preferences, with greater BOLD responses to sucrose than citric acid. Finally, we examined the influence of hydration level by contrasting BOLD responses to receipt of fluids when monkeys were thirsty and after ad libitum water consumption. BOLD responses in area $\mathrm{G}$ and area $12 \mathrm{o}$ in the left hemisphere were greater following full hydration. By contrast, BOLD responses in portions of medial frontal cortex were representing taste, taste preference and internal state.

Correspondence to: Elisabeth A. Murray, Ph.D., Laboratory of Neuropsychology, NIMH, Building 49, Suite 1B80, 49 Convent Drive, Bethesda, Maryland 20892-4415 USA, Ph: 301-443-7401, Fax: 301-402-0046, murraye@ mail.nih.gov.

Publisher's Disclaimer: This is a PDF file of an unedited manuscript that has been accepted for publication. As a service to our customers we are providing this early version of the manuscript. The manuscript will undergo copyediting, typesetting, and review of the resulting proof before it is published in its final citable form. Please note that during the production process errors may be discovered which could affect the content, and all legal disclaimers that apply to the journal pertain. 


\section{Keywords}

amygdala; ventral striatum; prelimbic cortex; orbitofrontal cortex; reward; motivation

\section{Introduction}

Several brain structures play a causal role in updating the value of expected and received rewards, based on current biological needs and context (Málková et al., 1997; Roberts et al., 2007; Rushworth et al., 2011; Murray and Rudebeck, 2018). The brain structures that play this causal role include the amygdala, the orbital prefrontal cortex (OFC), the medial prefrontal cortex (MFC), and related limbic structures. Behavioral and neurophysiological investigations intended to elucidate the brain mechanisms of reward processing and valuebased decision making in macaque monkeys typically employ behavioral tasks in which the monkeys are motivated to work for fluid or food rewards, often to satiety. Although reward value is fairly well understood in economic terms (e.g., subjective value is the product of reward magnitude and reward probability), the contribution of factors such as desirability of rewards and levels of motivation receive less attention. Gustatory stimuli vary not only in their sensory properties (e.g., dimensions of sweet, sour, bitter, salty, and umami), but also in their palatability. Accordingly, gustatory stimuli offer a means of interrogating the neural circuits that process these different kinds of information. Here we take advantage of the gustatory system and whole-brain functional magnetic resonance imaging (fMRI) to identify brain regions processing different aspects of subjective value, including taste, taste preference, and internal state. Our aims were to: 1) localize brain areas that encode taste; 2) identify brain areas sensitive to taste preference; and 3) establish which brain areas are sensitive to levels of hydration, which reflects internal state.

There is some uncertainty regarding the precise location and number of taste-related areas in macaque monkeys. One reason for the uncertainty is that single-unit recordings in macaques have found that only 2 to $5 \%$ of neurons in the anterior insula and overlying frontal operculum — in what is believed to be the primary gustatory cortex, area G — are taste responsive (Scott et al., 1986; Ogawa et al., 1989; Yaxley et al., 1990; Scott et al., 1991). Neurophysiological responses to taste extend beyond area G, and have been reported in somatosensory areas (Ogawa et al., 1989; Ito and Ogawa, 1994) and OFC (Rolls et al., 1990; Tremblay and Schultz, 1999; Pritchard et al., 2005; Ifuku et al., 2006; Padoa-Schioppa and Assad, 2006; Lara et al., 2009), a region contributing to value-based decision making (Padoa-Schioppa, 2011; Rudebeck and Murray, 2014). In addition, taste responses have been reported in ventrolateral prefrontal cortex area 12o, a region adjacent to OFC (Rolls et al., 1990). Our first aim was therefore to identify the full extent of taste-responsive cortex in macaques using fMRI. Based on prior work in taste sensory processing in macaques, we expected to find taste-responsive cortex in the anterior insular cortex and overlying frontal operculum, in the caudal portion of ventrolateral prefrontal cortex (area 12o), and in OFC area 13.

As a second aim, we assessed monkeys' relative preferences for three different fluids (sucrose, citric acid, and water), and examined fMRI data for correlates of taste preference. 
Though there is ample evidence that neurons in OFC, amygdala, ventral striatum (VS) and inferior temporal visual cortex (IT) are active in response to visual cues predicting fluid rewards, and encode the expected value of those rewards (OFC: Tremblay and Schultz, 1999; Roesch and Olson, 2004; Hosokawa et al., 2007; Morrison and Salzman, 2009; amygdala: Paton et al., 2006; Leathers and Olson, 2017; VS: Schultz et al., 1992; Hassani et al., 2001; Strait et al., 2016; IT: Mogami and Tanaka, 2006; Kaskan et al., 2017), less is known about the locations of neurons encoding the values of received tastes, or fluids in general (c.f. Bermudez and Schultz, 2010). In addition, most studies in macaques vary reward magnitude or probability as a means of manipulating value. Few have directly compared different tastes to evaluate subjective preferences that are inherent in the estimation of value and therefore influence choice behavior. In macaques, neurons encoding preference at the time of juice or food receipt have been reported in the OFC (Tremblay and Schultz, 1999), amygdala (Nishijo et al., 1986; Nishijo et al., 1988; Kadohisa et al., 2005) and the VS (Hassani et al., 2001; Cromwell et al., 2005). fMRI studies in humans likewise find BOLD responses correlated with the pleasantness of receipt of certain tastes (e.g., glucose, sucrose, monosodium glutamate) in OFC (Kringelbach et al., 2003; Small et al., 2003; Grabenhorst et al., 2008), amygdala (O’Doherty et al., 2001; Rolls et al., 2015) and striatum (Frank et al., 2008; Grabenhorst et al., 2008). MFC has also been reported to encode the pleasantness of certain tastes (McCabe and Rolls, 2007; Grabenhorst and Rolls, 2008; Rudenga and Small, 2013). We therefore predicted that BOLD activations reflecting taste preferences would be found in OFC, MFC, amygdala and striatum.

Finally, our third aim was to compare responses to freely delivered tastes before and after monkeys were given unlimited access to water. This 'hydration manipulation' was intended to reveal areas sensitive to changes in internal state. Although in the context of the present study ad libitum water is not equivalent to selective satiation, the two nevertheless share many influences. In the case of sensory-specific satiety, neurons in primary gustatory cortex have been reported to respond to tastes equally well before and after monkeys have been sated (Yaxley et al., 1988). By contrast, neurons in frontal cortex may increase or decrease their activity with satiety (Rolls et al., 1989; Pritchard et al., 2008; Bouret and Richmond, 2010). The activity of taste-responsive neurons in the amygdala likewise is modulated by satiety (Yan and Scott, 1996).

Some functional imaging studies in humans are consistent with reports that neurons in primary gustatory cortex are not affected by hydration using water (de Araujo et al., 2003), or satiety (Kringelbach et al., 2003). However, other studies report increased (Smeets et al., 2006; Haase et al., 2009; van Rijin et al., 2015) or decreased activation (rCBF, Small et al., 2001; Thomas et al., 2015; Eldeghaidy et al., 2016) in primary gustatory cortex with satiety. Reports of both increased (BOLD: de Araujo et al., 2003; Kringelbach et al., 2003) and decreased (rCBF: Small et al., 2001) activation have also been reported in secondary gustatory cortex with selective satiation, and decreases in rCBF have been reported as a function of satiety in the striatum, hypothalamus, temporal pole, and MFC (Gautier et al., 2000; Del Parigi et al., 2002; Small et al., 2003). fMRI results likewise demonstrate decreased activation in MFC with satiety (de Araujo et al., 2003; Smeets et al., 2006; Thomas et al., 2015). Based largely on these reports of brain areas with activations modulated by satiety, we predicted that BOLD responses in primary gustatory cortex area G 
would not reflect changes in the level of hydration, whereas BOLD responses in OFC, MFC, and the amygdala would be affected by changes in the level of hydration. Selective modulation of activity in OFC, MFC, and amygdala as a function of hydration state would affirm the role of these areas in indexing subjective value based on internal state, independent of task-related and somatosensory activity.

\section{Materials and Methods}

\subsection{Monkeys}

Three adult male rhesus monkeys (Macaca mulatta; weighing 4.7-7.1 kg at the beginning of training) were used in this study. Monkeys were pair housed when possible and kept on a 12$\mathrm{h}$ light-dark cycle with controlled access to water and periodic access to food during scheduled feeding times. All procedures were carried out in accordance with the Guide for the Care and Use of Laboratory Animals and were approved by the National Institute of Mental Health Animal Care and Use Committee.

\subsection{Behavioral Control Software}

MonkeyLogic (Asaad et al., 2008) was used to control both fMRI and the behavioral tasks, deliver taste stimuli, record eye position, record lick behavior, and to synchronize fMRI data with behavioral data. Each taste stimulus was delivered as a single $0.1 \mathrm{ml}$ bolus using an airpressurized system controlled with an electronic solenoid activated by a TTL pulse (Mitz, 2005). Volume delivery was calibrated by solenoid open times. Taste stimuli were delivered through an MRI-compatible device designed to deliver fluids via small tubes. A lick sensor was mounted on the monkey's chair and coupled to the fluid delivery tubes. Lick data were recorded via the displacement of the lick sensor (sampling frequency $1 \mathrm{kHz}$ ) using a Keyence fiber optic cable and custom-built electronics unit. In the scanner, lick sensor circuitry converted the sensor's displacement to an analog voltage, filtered the voltage to a bandpass of $1.7-340 \mathrm{~Hz}$, and provided a voltage gain that was adjusted each day to drive the $+/-10 \mathrm{~V}$ input range of the MonkeyLogic system. Eye position was monitored and filtered (4th order $350 \mathrm{~Hz}$ Bessel low pass filter) in the MRI scanner (iView eye-tracking system, SensoMotoric Instruments, SMI, sampling at 60 FPS) and in the training booth (Arrington ViewPoint EyeTracker, Arrington Research, Scottsdale, AZ, USA, sampling at 220 FPS).

\subsection{Taste Stimuli}

Two distinct tastes were used in all experiments: sucrose (sweet, 0.5M, pH 6.15, SigmaAldrich) and citric acid (sour, $20 \mathrm{mM}, \mathrm{pH}$ of 2.40, Sigma-Aldrich). Because of the difficulty of creating a tasteless solution for monkeys, we used distilled water (pH 6.10) as a third type of fluid. All water used to make taste solutions was pretreated via reverse osmosis and further purified by a HYDRO Picosystem Plus unit (Hydro Service and Supplies, Durham, NC, USA) containing one organic activated carbon adsorber, and one primary and one polishing mixed-bed deionizer. 


\subsection{General Training Procedures}

Each monkey was surgically implanted under aseptic conditions with a plastic fiberglassimpregnated head post while under isoflurane anesthesia. After recovery, monkeys were trained to sit in an fMRI-compatible chair, and were habituated to the training booth and to temporary restraint of head movement. Over the course of a few weeks, they were habituated to the MRI environment while wearing ear protection and undergoing head restraint.

\section{5. fMRI Data Acquisition}

fMRI data acquisition was carried out in the Neurophysiology Imaging Facility Core (NIMH, NINDS, NEI). Data were collected on a $4.7 \mathrm{~T}, 60-\mathrm{cm}$ vertical scanner (Bruker Biospec 47/60) with Bruker gradient coils $(6 \mathrm{Ga} / \mathrm{cm})$. A transmit coil $(20 \times 12.5 \mathrm{~cm})$, was placed over the caudal portion of each monkey's head. Left and right receive coils $(8.5 \times 7$ $\mathrm{cm}$ each) designed for optimal coverage of OFC, amygdala, and the striatum were positioned over the frontal lobes. Behavioral data (i.e., licking) were aligned to fMRI data using custom written software (MonkeyLogic, MATLAB) by recording and counting volume pulses, or TRs, from the scanner.

In each session, shimming was carried out using a field map measurement. Single-shot echo planar images (EPI) were acquired with parameters optimized for BOLD imaging: echo time (TE), $22 \mathrm{~ms}$; flip angle, $76^{\circ}$; repetition time (TR), $2.2 \mathrm{~s}$; matrix size $96 \times 48 \mathrm{~mm}$ coronal; voxel size, $1.5 \mathrm{~mm}^{3}$ isotropic.

The taste localizer task made use of a 'free-reward' design whereby monkeys received one of three fluids in random order, without predictive cues. Following the start of a trial, which was unsignaled, a one-second interval preceded the delivery of one of the three fluids, followed by 5 seconds of fMRI and lick data collection before the end of the trial. An intertrial interval was chosen from a standard uniform distribution of 2 to 8 seconds according to a pseudorandom order. Lick behavior was recorded by measuring the displacement of the MRI compatible fluid delivery assembly attached to monkeys' chairs.

Acquisition of fMRI data occurred over the course of 10 months. We acquired two sets of scans; each monkey received 5 scan sessions before and 5 sessions after being fully hydrated (i.e., before and after ad libitum access to water for 5 days in monkey 1, and 3 days in monkeys 2 and 3 ). The average duration between scans was 4.5 days. Monkeys sat quietly in a MR-compatible primate chair while they passively received fluids. During each scan session before being fully hydrated, monkeys received an average of $539.93+/-31.35$ trials $(181.73+/-11.14$ water trials, $181.67+/-10.82$ sweet trials, and $176.53+/-10.36$ sour trials). In scan sessions after full hydration, monkeys received an average of $487.33+$ / -20.41 trials $(161.33+/-7.36$ water trials, $162+/-7.63$ sweet trials, and $162+/-6.76$ sour trials).

The number of runs per fMRI session was as follows: Monkey 1: Thirsty: 8, 12, 11, 11, 8; Hydrated: 8, 8, 8, 8, 8; Monkey 2: Thirsty: 4, 12, 12, 7, 12; Hydrated: 10, 10, 5, 10, 10; Monkey 3: Thirsty: 12, 10, 10, 9, 10; Hydrated: 10, 10, 10, 10, 10. 
For all three monkeys, we measured the temporal signal-to-noise ratio (tSNR) in each of the five scans sessions collected before (Thirsty) and after (Hydrated) free access to water, and created an average tSNR map. tSNR values were calculated from residuals after regression. Given that our typical imaging session contained on average 2730 time points, we determined that an appropriate tSNR cutoff value would be 40, based on empirical data and models (Murphy et al., 2007); this estimate is consistent with previous work (Simmons et al., 2010). Although tSNR maps in our prior study with the same monkeys and equipment showed good signal acquisition throughout the brain (Kaskan et al., 2017), some brain regions in the present study-specifically, the entorhinal and perirhinal cortex, medial amygdala and OFC - showed lower tSNR values. This was likely due to differences in the experimental design that affected the total amount of explained variance, prior to tSNR calculations.

\subsection{Choice Preferences and Lick Analysis}

Taste preferences were identified by requiring monkeys to perform a modified Choice and View task (Kaskan et al., 2017) in a training booth outside the scanner. These sessions were administered on nonscan days. Three simple shapes (a pentagon, an "X", and an "O") predicted the delivery of fluid. Each shape was assigned to one each of the three fluids, and shape-fluid assignments differed across monkeys. The task consisted of interleaved twoalternative forced-choice trials and view only trials. On Choice trials, following $250 \mathrm{~ms}$ of initial fixation on a spot within a $3^{\circ}$ window, two cues were presented $7.5^{\circ}$ from the center of the screen. The monkey was required to maintain central fixation for an additional $250 \mathrm{~ms}$ before making a saccade to one of the two cues. Once the monkey had made a saccade, as determined by the eye position entering an $11^{\circ}$ window surrounding the chosen cue, the cue not chosen was removed from the screen. The monkey was then required to maintain gaze within the chosen cue fixation window for $750 \mathrm{~ms}$. Upon successful completion of this fixation requirement, the chosen cue was removed, and after a pseudorandomly determined delay chosen from a standard uniform distribution of 2 to 4 seconds, the associated taste was delivered to the monkey. Following fluid delivery, an intertrial interval pseudorandomly chosen from a standard uniform distribution of 2 to 8 seconds was initiated.

On View trials, following $250 \mathrm{~ms}$ of initial fixation on a spot within a $3^{\circ}$ window, a single cue was presented in the center of the screen. The monkey was required to maintain gaze within an $11^{\circ}$ window surrounding the centrally placed cue. Upon completion of this gaze requirement, the cue disappeared; a pseudorandomly determined delay chosen from a standard uniform distribution of 2 to 4 seconds preceded the delivery of the associated taste.

We collected two measures of each monkey's taste preference from the Choice and View task: choices and anticipatory licking. To assess monkeys' choice preferences for cues associated with delivery of sucrose, citric acid and water, we counted each instance the monkeys chose a cue predicting the delivery of a particular fluid out of the total number of Choice trials in which two specific options were offered (e.g., the number of sucrose cues chosen divided by the number of sucrose vs. water choice trials). Choices were collapsed across days and results were expressed as a percent of fluid type chosen for each possible pair of fluids. Preferences were determined using a two-tailed binomial test. 
Anticipatory licking was evaluated on View trials. We recorded anticipatory licking behavior from cue onset to the earliest possible time of fluid delivery, a period of 3 seconds. Lick traces were mean-shifted by the day's mean to account for differences in mechanical setup each day, and rectified (absolute value). We created an area under the curve (AUC) measurement for each trial. The average AUC for sucrose, citric acid, and water was plotted \pm 1 SEM to compare licking between each taste for a given session, for a given monkey. To test for significant differences in anticipatory licking to cues predictive of each of the fluids, a two-sample t-test was used to test anticipatory licking for each monkey for each session. Additionally, we ran a fixed-effects ANOVA with factors of monkey, fluid, and session, with session nested under monkey, to assess population-level effects.

Although we attempted to assess monkeys' choice preferences and anticipatory licking after monkeys were allowed ad libitum access to water, we were unable to collect enough trials to reliably ascertain any preferences.

Licking was also measured during fMRI. To determine the times monkeys licked, we measured the mechanical displacement of an optical lick sensor. We determined the times at which displacement crossed 10 percent of the maximum displacement on each trial in order to: 1) determine lick times prior to fluid delivery (which served as nuisance regressor; 1 of 4 regressors in a general linear model (GLM), below); and 2) determine the times monkeys first licked in response to the delivery of each of the three fluids ( 3 of 4 regressors for the GLM of fMRI data, one regressor for each fluid type). The first time this threshold was exceeded prior to fluid delivery, the time was classified as 'lick time prior to fluid delivery'. The first time this threshold was exceeded after the fluid solenoid was opened, the time was classified as 'fluid delivery time' for each of the three types of fluids.

Finally, to assess potential changes in licking behavior that may have occurred as a result of monkeys having received water, we quantified licking to the receipt of each of the fluids in the scanner before and after monkeys were given water ad libitum. Specifically, we used an AUC measurement (detailed above) in a mixed-effects ANOVA with monkey, fluid, hydration status, and fMRI run as fixed effects, and session as a random effect, with session and hydration status each nested under monkey, and run nested under session.

\section{7. fMRI Data Analysis}

Preprocessing was carried out using AFNI (Analysis of Functional NeuroImages, NIMH; Cox, 1996) on raw data in native space prior to regression and alignment. Runs were PLACE corrected to compensate for geometric field distortions (Xiang and Ye, 2007), slice-time corrected, and resampled in pitch from $22^{\circ}$ to plumb (de-obliqued). To allow the scanner to reach a steady state, the first 5 volumes of each run were removed; the data were concatenated across runs within a single session, motion corrected using volume registration, and blurred to $2 \mathrm{~mm}$. Anatomical scans (MDEFTs) in native space from each monkey from each session were manually skull stripped and aligned to a high-resolution skull-stripped scan from our monkey template (monkey 3) using a 12-dimension affine transformation. Spatial transformation matrices for aligning anatomical scans to our monkey template for each session were applied to preprocessed native-space statistical data (preprocessed raw data after regression) for group analysis. fMRI data are therefore 
presented on our template macaque brain, and may not match precisely sections shown in an atlas.

As indicated earlier, licks in response to the delivery of each fluid type were used to identify fluid delivery times for regression. Lick times were deconvolved with tent functions in AFNI to derive estimates of the hemodynamic response time course for each type of fluid. In a separate analysis, regressors of interest (licks resulting from fluid delivery) and those occurring prior to fluid delivery (nuisance regressors) were convolved with a standard 'boxcar' hemodynamic response function (HRF) and entered into a GLM to obtain regression coefficients for each taste and self-initiated licks (to create an exclusion mask, see below). Regression coefficients for each scan were then entered into a mixed-effects ANOVA (3dLME in AFNI; $z$-stats reported) with monkey nested in session, and session treated as a random factor, to generate contrast maps of the different fluid types. We note that many fMRI studies in humans use each subject as a data point, and, to get enough power, experiments may need upwards of 15 subjects. Alternatively, in some fMRI studies with nonhuman primates, data are pooled across sessions and each time point (volume) is used as a data point. Our analysis is a compromise between these two approaches; we use each session in each monkey as a data point in a second-level analysis.

To identify taste-responsive areas we used the contrast: ( 0.5 sucrose +0.5 citric acid $)$ / water (3dANOVA in AFNI; $t$-stats reported). By dividing the response to sucrose and citric acid by water, we were able to normalize taste responses with responses resulting from the general effect of fluid in the mouth and swallowing.

All maps were initially thresholded at a $p<0.001$ uncorrected. A cluster size threshold of 8 voxels was used to control the familywise error rate (FWE) at $p<0.01$ in all maps shown. The cluster size ( 8 voxels) corresponded to the $99.9 \%$ quantile of the distribution of the maximum cluster size simulated via 10,000 Monte Carlo stimulations (Forman et al., 1995), using estimates of spatial smoothness based on the residuals of the GLM.

To examine the development of the hemodynamic response, BOLD time-course plots were generated. Where illustrated, time-course plots show the average percent signal change for all voxels in the cluster.

BOLD responses associated with licking in the absence of fluid delivery were used to further mask responses associated with somatosensory or motor components of licking and associated orofacial movements. The mask, created from the contrast of self-initiated licks vs. baseline, thresholded at $p=0.01$ (uncorrected), was inverted to contain zero-value voxels at locations which had a response to licking prior to fluid delivery, and values of one elsewhere. An algebraic sum of taste responses ( $(0.5$ sucrose +0.5 citric acid $) /$ water $)$ and this mask created from the nuisance regressor of licks prior to fluid delivery excluded voxels activated in response to licking prior to fluid delivery.

To identify brain areas with activations modulated by taste preference we used the contrast: sucrose minus citric acid. The underlying assumption is that each fluid has a strong (and roughly equivalent) taste component. In addition, the fluids involved equivalent amounts of licking and swallowing. Thus, although differences in sensory properties cannot be ruled 
out, the activation observed in the sucrose minus citric acid contrast likely reveals subjective preference.

To identify brain areas in which activations changed as a function of hydration state we performed a whole-brain analysis using the contrast: all fluids before monkeys had received ad libitum water minus all fluids after monkeys had received ad libitum water. To illustrate the data we created a mask of taste-responsive areas (described above; 0.5 sucrose +0.5 citric acid) / water) and present BOLD signal changes within the taste-responsive areas, as well as outside of those areas.

Finally, in order to characterize the locus of activation with respect to defined areas and regions (e.g., insula), we cite the primary literature in defining and localizing cortical areas where relevant, and make use of a macaque brain atlas (Saleem and Logothetis, 2012) for reference.

\section{Results}

During fMRI scan sessions, monkeys received small amounts of three different fluids, delivered one at a time, in pseudorandom order: sucrose, citric acid, and water. The pattern of BOLD responses during the passive receipt of fluids was used to identify taste-responsive brain areas. Fluid preferences were assessed outside the scanner using two measures: behavioral choice and anticipatory licking. Preference measures were related to BOLD responses to determine whether brain regions signaled taste preference. Finally, manipulations of hydration status were used to determine whether BOLD responses to taste were modulated by changes in internal state.

\subsection{Gustatory Responses Identified with fMRI}

To identify brain areas responsive to taste, we contrasted BOLD responses to sucrose and citric acid with BOLD responses to water. Detecting licks made in the absence of fluid delivery and their associated BOLD responses allowed us to mask lick-related responses after receipt of fluid. Thus, activations related to the sensorimotor aspects of licking were controlled for in this analysis, revealing activations related to tastes.

Areas exhibiting greater BOLD responses to sucrose and citric acid than water are shown in Figure 1. Areas identified in this contrast include primary gustatory cortex area G, area 12o, and the anterior insula. Two distinct clusters were identified in the left hemisphere. One cluster ("Cluster 1", Fig. 1A-D), comprised of 24 voxels, extended $6 \mathrm{~mm}$ in the anteriorposterior (AP) dimension from area $12 \mathrm{o}$ to area $\mathrm{G}$ to the anterior and dorsal portion of the insula. A second cluster ("Cluster 2", Fig. 1E-H), caudal to the first, comprised 17 voxels, and spanned $6 \mathrm{~mm}$ in AP extent along the anterior insular cortex adjacent to the superior limb of the limiting sulcus. In the right hemisphere, a single cluster of 36 voxels was identified in roughly the same region as the more caudal cluster in the left hemisphere. The anterior voxels comprising this single right hemisphere cluster were located in area $G$ and extended $7.5 \mathrm{~mm}$ AP into the superior limb of the limiting sulcus to include the neighboring anterior insula. Coronal sections shown in Figure 1 are spaced at $1.5 \mathrm{~mm}$, the size of our functional voxels. 
In MFC area 25 we identified another distinct cluster, comprised of 17 voxels; unlike the voxels in area $\mathrm{G}$, area $12 \mathrm{o}$ and the anterior insula, those in area 25 exhibited greater BOLD responses to water than to sucrose and citric acid.

\subsection{Licking Responses Identified with fMRI}

Unmasked taste responses (sucrose and citric acid vs. water) are presented in Supplemental Figure 1 in order to demonstrate areas activated by licking (and, potentially, by taste as well). Licking led to prominent activations at sites relatively distant from the taste-response zones illustrated in Figure 1, in the depths of the central sulcus and more rostral sites on the lateral suface. These sites are presumed to be the face and oral cavity representations in somatosensory cortex areas 3a, 3b, 1 and 2 (Supplemental Figure 1, Sections I-O). Activation of motor cortex was more limited, and involved the face representation of primary motor cortex (face-MI) and / or the cortical masticatory area (Huang et al., 1989) (Supplemental Figure 1, Section H).

\subsection{Taste Preferences as Revealed by fMRI, Behavioral Choice, and Anticipatory Licking}

We used monkeys' choice preferences and anticipatory licking in the presence of fluidpredictive cues to assess fluid preferences. These data were collected during three test sessions conducted outside the scanner after fMRI data had been acquired for the first set of scans (see Methods 2.5), prior to offering monkeys water ad libitum. We then examined fMRI data with respect to monkeys' preferences to determine if any areas exhibited greater BOLD signals to preferred fluids.

In the fMRI analysis, the contrast of sucrose vs. citric acid did not localize any voxels in taste-responsive areas G, 120 or the insular cortex; instead, this contrast revealed voxels in an area of the striatum that receives inputs from the OFC (hereafter called the ventral striatum, VS), extending into the dorsal and ventral pallidum and left anterior amygdala. Figure 2 shows the sucrose vs. citric acid contrast at three different statistical thresholds, masked with licking in the absence of fluid delivery. One large cluster of 66 voxels spanned the midline and extended roughly $9 \mathrm{~mm}$ in AP extent, from the VS to the ventral pallidum, paraventricular nucleus of the hypothalamus (PVN), and medial preoptic area (MPOA) at more posterior levels. A second cluster of 19 voxels extended $6 \mathrm{~mm}$ in AP extent from the medial aspect of the temporal pole (TGa) to the rostral portion of the lateral and basal nuclei of the amygdala in the left hemisphere. The contrast of sucrose vs. citric acid unmasked for licking prior to fluid delivery is presented in Supplemental Figure 2. Masking with licking prior to fluid delivery did not affect the finding that BOLD responses in the amygdala and VS were greater for sucrose relative to citric acid.

We tentatively identified the voxels in this contrast as reflecting taste preference. To assess taste preferences, we offered each monkey pair-wise choices among the three fluids. In Figure 3A, monkeys' choice preferences are plotted as a percentage of the number of times a given cue was chosen out of the total number of trials on which a given pair of fluids was on offer: sucrose / (sucrose vs. water); sucrose / (sucrose vs. citric acid); and citric acid / (citric acid vs. water). All three monkeys strongly preferred sucrose to both citric acid and water 
(all $p s<0.001$ ). All three showed less marked preferences for the trials with water vs. citric acid.

In addition to measuring choice preferences, anticipatory licking during presentation of single cues (View trials) was evaluated to get an ancillary measure of monkeys' preferences. In Figure 3B, monkeys' mean anticipatory lick behavior is plotted ( \pm 1 SEM) as an AUC measurement for each trial and averaged for each fluid type per session. Only monkeys 2 (sucrose $>$ water, $p=0.026$ ) and 3 (sucrose $>$ water, $p=0.024$; sucrose $>$ citric acid, $p=$ 0.018 ) licked more for sucrose cues, and only in one session each; monkey 1 showed no anticipatory lick preferences to any taste cues. Although anticipatory licking did not provide as robust a measure of preference as monkeys' choices, it nevertheless was consistent with choice behavior. Given the foregoing, we conclude that the greater BOLD responses to sucrose than citric acid in the VS, ventral pallidum, and TGa/amygdala, as shown in Figure 2 , likely reflect taste preference and/or subjective value.

\subsection{Internal State Changes Identified with fMRI}

Finally, we wondered whether we could reveal influences of hydration status on signals related to taste or taste preference. fMRI scans were acquired from the same three monkeys using the same schedule as before, but this time after they had been allowed ad libitum access to water for 3-5 days. Although we conducted a whole-brain analysis, we show the effects of changes in hydration in two figures, the first restricted to taste-responsive areas (Figure 4), defined by a mask (see Methods), and the second restricted to areas outside the taste-responsive zones (Figure 5).

We first consider the effects of hydration status in taste-responsive areas. Only one cluster within taste-responsive cortex was modulated by hydration: voxels resided in area 12o, area $\mathrm{G}$, and the anterior insular cortex in the superior limb of the limiting sulcus in the left hemisphere (Figure 4). This single cluster spanned $9 \mathrm{~mm}$ in AP extent and comprised 28 voxels. No taste responsive voxels in the right hemisphere exhibited hydration effects at $\mathrm{p}=0.001$ (FWE corrected $\mathrm{p}=0.01$ ), nor were significant voxels evident at lower thresholds of $\mathrm{p}=0.01$ or $\mathrm{p}=0.005$ (FWE corrected $\mathrm{p}=0.01$ ). The effects of hydration were nonspecific for the tastes used; there was no interaction between fluid type and hydration. Given the lateralized finding of the effect of hydration (area G, area 12o, left hemisphere) we tested for hemispheric differences by contrasting data in original space to that in a "mirror-image" space (i.e. standard space vs. left-right flipped). We did not detect any significant differences between hydration effects in the left vs right hemispheres; thus, there was no evidence for hemispheric specialization of the hydration effects.

We looked beyond taste-responsive cortex to identify other areas that might exhibit altered BOLD responses to all fluids as a result of monkeys consuming water ad libitum. Figure 5 illustrates a large cluster in MFC localized to area 32 and the adjacent portion of area 10. In this region, greater BOLD responses were found before relative to after hydration (i.e., thirsty > hydrated; red-orange color scale). Similarly, clusters in the right and left anterior dorsal striatum exhibited greater BOLD responses prior to hydration. A third single cluster that included the anterior insula in the right hemisphere and the perirhinal cortex in the right 
temporal pole exhibited greater BOLD responses following water ad libitum (i.e., hydrated > thirsty; blue color scale).

To assess whether the foregoing effects on BOLD activations could be accounted for by changes in licking behavior, we quantified licking in the scanner before and after manipulations of hydration using a mixed-effects ANOVA that specified monkey, fluid, hydration status, and fMRI run as fixed effects, and session as a random effect, with session and hydration status each nested under monkey, and run nested under session. We found significant main effects of monkey $\left(\mathrm{F}_{2,857}=4.77, p=0.030\right)$ and fluid $\left(\mathrm{F}_{2,857}=21.84, p=\right.$ $\left.3.87 \times 10^{-6}\right)$, but no effect of hydration status $\left(\mathrm{F}_{3,857}=3.30, p=0.058\right)$, indicating that monkeys' licking was not altered due to changes in hydration. Thus, the different pattern of BOLD responses found before and after hydration cannot be accounted for by changes in licking behavior.

\section{Discussion}

\subsection{Primary Gustatory Cortex in Macaques}

To our knowledge, this is the first functional imaging study of the gustatory system in macaque monkeys to make use of event-related BOLD methodology. Although an eventrelated task design allowed for the inclusion of trial-by-trial licking estimates in the model, its reduced power relative to a block design limited our analysis to group level effects (see Section 2.7). Nonetheless, this adequately revealed effects in our sample of three monkeys. We used whole-brain BOLD fMRI to overcome the spatial limitations inherent in singleneuron recording studies of taste-related areas. Despite the small numbers of taste responsive neurons reported in primary gustatory cortex (area $\mathrm{G}$ ) of macaques ( 2 to 5\%: Scott and Mark, 1986; Ogawa et al., 1989; Yaxley et al., 1990; Scott et al., 1991), we were able to identify robust BOLD responses in this region (Figure 1B-D). Although we were not able to assess cytoarchitectonic borders, the cortex we identified as taste responsive most certainly includes area G, based on regional landmarks (Saleem and Logothetis, 2012).

Consistent with our finding of taste-related activations in area $\mathrm{G}$ and the anterior insula, anatomical studies have reported projections from the primary gustatory relay nucleus of the thalamus, VPMpc, to the anterior insular cortex and overlying frontal operculum (Kusama et al., 1985; Pritchard et al., 1986; Ogawa, 1994). In addition, taste-related activations in area $12 \mathrm{o}$ are consistent with the finding that this region of frontal cortex receives direct projections from area $\mathrm{G}$ and anterior insula (Baylis et al., 1995). Although early anatomical studies also reported projections from thalamic nucleus VPMpc to somatosensory cortex (Kusama et al., 1985; Pritchard et al., 1986; Ogawa, 1994) more recent neurophysiological mapping studies (Cerkevich et al., 2013) found no projections from VPMpc to somatosensory areas $3 \mathrm{a}, 3 \mathrm{~b}, 1$ or 2 . The latter study suggests a more limited projection from VPMpc to primary gustatory cortex. Although use of the licking mask allows us to be confident that the taste-responsive regions we observed in area G, 12o and anterior insula are not an artifact of differences in licking between conditions, it complicates other aspects of the interpretation. Specifically, use of the licking mask prevents firm conclusions regarding activations in areas 3a, 3b, 1 and 2 and M1. One possibility is that these sites are involved 
with sensorimotor aspects of gustation (e.g., licking) only. Alternatively, they may be involved in both taste and licking.

\subsection{Taste-Responsive Areas Beyond Primary Gustatory Cortex}

Consistent with single-unit neurophysiological mapping studies in macaques, we identified taste-responsive regions not only in insular cortex and frontal operculum, but also ventrolateral prefrontal cortex area $12 \mathrm{o}$, a region identified as secondary gustatory cortex (Rolls et al., 1990).

Despite the occurrence of taste-related neuronal activity in area 13 (Rolls et al., 1990; Tremblay and Schultz., 1999; Pritchard et al., 2005; Ifuku et al., 2006; Padoa-Schioppa and Assad, 2006; Lara et al., 2009), we did not find robust BOLD responses in this region. Our failure to identify area 13 as taste-responsive cortex could be due to mixed patterns of neuronal activity (increases in firing rates to a particular fluid in some neurons, and decreases in firing rates in other neurons). Alternatively, perhaps the neuronal activity in some neurophysiological investigations reflects particular tastes interacting with different types of task-related activity (choice, action planning). Finally, we cannot rule out the possibility that signal dropout in this region led to a null result.

\subsection{Brain Regions Encoding Taste Preference}

By contrasting BOLD responses to sucrose to those from citric acid we aimed to identify areas signaling taste preference. The underlying assumption is that each fluid has a strong (and roughly equivalent) taste component, allowing activation related to subjective preference to emerge. This contrast revealed greater BOLD responses for sucrose than citric acid in the amygdala, VS, and ventral pallidum. Three findings support our contention that these regions encode subjective taste preference. First and foremost, monkeys showed a strong choice preference for sucrose and, to a much lesser extent, increased anticipatory licking for sucrose, mirroring the fMRI findings. Second, the same contrast does not yield brain areas engaged in basic gustatory processing, and, conversely, taste-responsive areas identified by our taste localizer do not exhibit greater BOLD responses to sucrose than citric acid. Third, a corollary to the second, neither the amygdala nor VS was activated by our taste localizer.

It is somewhat surprising that preference effects were not found in area 13, where the activity of many neurons reflects taste preference (Tremblay and Schultz, 1999; PadoaSchioppa and Asaad, 2006), and magnitude of reward (Roesch and Olson, 2004; Kennerley and Wallis, 2009; Morrison and Salzman, 2009; Rudebeck et al., 2013). Still, negative findings are not meaningful for the same reasons given before; mixed increases and decreases of the activity of single neurons could well cancel out BOLD responses related to taste preference, and we cannot rule out the possibility of signal dropout in this region.

4.3.1. Amygdala.-Our fMRI findings of activations related to taste preference (e.g., contrast of sucrose vs. citric acid) in the amygdala are in agreement with the few studies examining neuronal correlates of taste preference in macaque monkeys. Specifically, neurons in the amygdala are more active during ingestion of preferred foods and preferred 
complex tastes relative to nonpreferred ones (Nishijo et al., 1988; Kadohisa et al., 2005). In humans, PET and fMRI studies find greater amygdala activation during processing of highly palatable and aversive tastes (Zald et al.,1998; O'Doherty et al., 2001), suggesting the amygdala is involved in processing either tastes or their associated valence or both.

4.3.2. Ventral striatum.-In the present study, activations in the VS appeared to reflect taste preference. Many studies in humans report that the striatum encodes taste pleasantness at the time of fluid delivery. For example, one study found the pleasantness of fat in the mouth correlated positively with the BOLD response in the VS (Grabenhorst et al., 2010), and another found greater activations in the VS for sucrose relative to an artificial sweetener (sucralose), which subjects found less pleasant than sucrose (Frank et al., 2008). To the extent that pleasantness reflects preference, our findings fit with these reports.

4.3.3. Interpretational limitations.-It is possible that differences in sensory features contributed to the differences in activation arising from the sucrose vs. citric acid contrast either in addition to, or instead of, preference per se. Future studies should include additional tastes and gradations of preference to test the generality of activations related to taste preference. In this study, we used tastes we expected to be palatable or neutral. Future studies might evaluate responses to a variety of palatable and aversive tastes over a range of concentrations to allow identification of brain areas encoding taste preference vs. taste valence independent of preference.

\subsection{Brain Regions Reflecting Changes in Internal State}

As a means of further characterizing taste related areas, we compared activations to passive receipt of fluids before and after monkeys had free access to water. This allowed us to contrast brain responses, within subjects, to receipt of fluids in thirsty versus hydrated states. The free access to water was not intended to induce a selective satiation, but, rather, to alter the overall level of hydration which should affect neural circuits sensitive to internal state. Unsurprisingly, one effect that accompanied the free access to water was a change in the monkeys' willingness to work for fluids. When thirsty monkeys were given the Choice and View task outside the scanner, they routinely chose taste-predictive cues to earn fluid rewards. After monkeys had received full access to water for 3-5 days, they refused to choose taste-predictive cues, indicating they had lost the motivation to work for fluid rewards.

There is a large literature on single neuron responses in macaques to selective satiety, though not one for hydration per se. As mentioned earlier, we did not induce a state of selective satiation. Nevertheless, selective satiety and hydration likely share many effects, so we discuss each condition as appropriate.

Although it is widely believed that satiety produces a decrease in neuronal firing rates in many frontal cortex areas (Rolls et al., 1989; Pritchard et al., 2008), recent studies have revealed a more complex pattern. For example, some frontal cortex areas contain a mixture of neurons, some that increase and others that decrease their firing rates with satiety (Bouret and Richmond, 2010); some neurons change their activity only after certain feeding intervals (Rolls et al., 1988). Within the taste-responsive areas, primary gustatory cortex in the left 
hemisphere exhibited positive trending BOLD responses following hydration. Interestingly, Rolls et al. (1988) report that consumption of fluids to satiety in monkeys failed to alter neural responses in primary gustatory cortex, mirroring the lack of hydration effects we report for the right hemisphere. In future studies it will be important to take into account the hemisphere from which neurons are recorded. Small and colleagues (Small et al., 2001), however, did report reduced regional blood flow in primary gustatory cortex in humans as they became sated on chocolate. Yet we find that BOLD responses in primary gustatory cortex in the left hemisphere are greater following hydration, an effect opposite in sign to what would be expected from the findings of Small et al (2001). Perhaps one of two antagonistic populations decreased firing rates as monkeys became hydrated, leaving the other active; our experimental design and technique precludes observing these types of changes. Alternatively, perhaps selective satiation and hydration have fundamentally different influences on taste processing in primary gustatory cortex.

As for higher-order gustatory areas, we find hydration effects in area $12 \mathrm{o}$ in the left hemisphere. This area did not respond when monkeys were thirsty, but did exhibit positive trending BOLD responses when monkeys were hydrated. A neurophysiological study in macaques found neurons in ventrolateral prefrontal cortex area 12o decrease their firing rates with satiety (Rolls et al., 1989). These authors argued that primary areas encode sensory aspects of taste, whereas higher-order areas may encode the motivational properties of tastes. Although we do find that activations in area 120 in the left hemisphere are modulated by changes in hydration, this area exhibits greater BOLD responses in the hydrated relative to the thirsty state, which is the opposite direction that one would predict based on the single neuron recordings. As mentioned earlier with respect to primary gustatory cortex, one population of neurons with opposing activity to another could have decreased firing with hydration, driving a positive trending BOLD response only after monkeys had consumed water to satiety. Another possible explanation for increased BOLD responses following free access to water is that some neurons may develop taste responses; for instance, in OFC areas 11 and 13, some neurons become responsive to tastes with satiation (Pritchard et al., 2008).

Outside taste-responsive areas, we identified MFC areas 32/10m as having reduced BOLD responses in the hydrated vs. thirsty state. Consistent with this observation, neuronal responses in MFC decrease as monkeys work to satiety for water (Bouret and Richmond, 2010). Several reports from studies in humans likewise fit with our finding of decreased BOLD responses in MFC following ad libitum consumption of water. For instance, regional cerebral blood flow (rCBF) has been reported to decrease in MFC as subjects ate chocolate to satiety (Small et al., 2001), after the consumption of a meal (Del Parigi et al., 2002; Thomas et al., 2015), and after consumption of chocolate milk (Smeets et al., 2006). Most pertinent to the present study, as was the case in our monkeys given water ad libitum, humans given water to satiety show decreased BOLD responses in MFC (de Araujo et al., 2003).

One possibility suggested by these findings is that area $32 / 10 \mathrm{~m}$ is involved with motivational aspects of received fluids, a concept consistent with the idea that expected value is represented in a common currency (Kable and Glimcher, 2007) or that value information is 
integrated in this region (Suzuki et al., 2017). In addition, this region is one of several anatomically related regions implicated in motivated behavior; as such, it potentially provides insight into the neural mechanisms underlying apathy and anhedonia (Husain and Roiser, 2018).

Notably, the MFC areas 32/10m appeared to be much more sensitive to changes in hydration status than to fluid preference, per se. Consistent with this interpretation, although preference and willingness to work for a food or fluid often vary together, there are many instances in which the two factors can be dissociated (e.g., liking vs. wanting, see review by Berridge and Kringelbach, 2015). Against this interpretation, however, being in a hydrated vs. thirsty state likely induces a number of effects on brain activity (e.g., related to arousal, stomach distension, mouth dryness, etc.) that are not contolled for in the present study. In addition, because we used a pre-post hydration contrast, we cannot rule out the possibility of order effects. Future studies should address how factors related to reward desirability, availability, and internal state are combined in MFC to influence choice.

\subsection{Summary}

To summarize, we leveraged the gustatory system to highlight brain areas associated with taste, taste preference and internal state. In most circumstances subjective value and internal states related to satiety vary together, and are therefore confounded. Here, we were able to dissociate these factors to some extent, at least neurally. With respect to our original aims, we: 1) localized brain areas encoding taste; 2) identified areas sensitive to taste preference; and 3 ) established brain areas sensitive to changes in hydration state, some of which may reflect changes in motivation. Our fMRI investigation in nonhuman primates allowed for a within-subjects repeated measures design to uncover information about the neural correlates of taste, preference, and internal state that might not have been possible in human studies.

\section{Supplementary Material}

Refer to Web version on PubMed Central for supplementary material.

\section{Acknowledgements}

This research was supported by the Intramural Research Program of the NIMH (EAM, ZIAMH002886) and a NARSAD Young Investigator Award from the Brain and Behavior Research Foundation (PMK). We thank the staff of the Neurophysiological Imaging Facility, of the NIMH Scientific and Statistical Computing Core, and of the NIMH Section on Instrumentation for their invaluable assistance. Finally, we thank Maia Pujara, Jason Avery, and Vincent Costa for comments on an earlier version of this manuscript.

\section{References}

Asaad FW, Emad N, Eskandar EN, 2008 A flexible software tool for temporally-precise behavioral control in MATLAB. J Neurosci Methods 174, 245-258. [PubMed: 18706928]

Baylis LL, Rolls ET, Baylis GC, 1995 Afferent connections of the caudolateral orbitofrontal cortex taste area of the primate. Neuroscience 64, 801-812. [PubMed: 7715788]

Bermudez MA, Schultz W, 2010 Responses of amygdala neurons to positive reward-predicting stimuli depend on background reward (contingency) rather than stimulus-reward pairing (contiguity). $\mathbf{J}$ Neurophysiol 103, 1158-1170. [PubMed: 20032233] 
Berridge KC, Kringelbach ML, 2015 Pleasure systems in the brain. Neuron 86, 646-664. [PubMed: 25950633]

Bouret S, Richmond BJ, 2010 Ventromedial and orbital prefrontal neurons differentially encode internally and externally driven motivational values in monkeys. J Neurosci 30, 8591-8601. [PubMed: 20573905]

Cerkevich CM, Qi HX, Kaas JH, 2013 Thalamic input to representations of the teeth, tongue, and face in somatosensory area $3 b$ of macaque monkeys. J Comp Neurol 521, 3954-3971. [PubMed: 23873330]

Cox RW, 1996 AFNI: Software for analysis and visualization of functional magnetic resonance neuroimages. Comp Biomed Res 29, 162-173.

Cromwell HC, Hassani OK, Schultz W, 2005 Relative reward processing in primate striatum. Exp Brain Res 162, 520-525. [PubMed: 15754177]

de Araujo IE, Kringelbach ML, Rolls ET, McGlone F, 2003 Human cortical responses to water in the mouth, and the effects of thirst. J Neurophysiol 90, 1865-1876. [PubMed: 12773496]

Del Parigi A, Chen K, Salbe AD, Gautier JF, Ravussin E, Reiman EM, Tataranni PA, 2002 Tasting a liquid meal after a prolonged fast is associated with preferential activation of the left hemisphere. Neuroreport 13, 1141-1145. [PubMed: 12151757]

Eldeghaidy S, Marciani L, Hort J, Hollowood T, Singh G, Bush D, Foster T, Taylor AJ, Busch J, Spiller RC, Gowland PA, Francis ST, 2016 Prior Consumption of a Fat Meal in Healthy Adults Modulates the Brain's Response to Fat. J Nutr 146, 2187-2198. [PubMed: 27655761]

Forman SD, Cohen JD, Fitzgerald M, Eddy WF, Mintun MA, Noll DC, 1995 Improved assessment of significant activation in functional magnetic resonance imaging (fMRI): use of a cluster-size threshold. Magn Reson Med 33, 636-647. [PubMed: 7596267]

Frank GK, Oberndorfer TA, Simmons AN, Paulus MP, Fudge JL, Yang TT, Kaye WH, 2008 Sucrose activates human taste pathways differently from artificial sweetener. Neuroimage 39, 1559-1569. [PubMed: 18096409]

Gautier JF, Chen K, Salbe AD, Bandy D, Pratley RE, Heiman M, Ravussin E, Reiman EM, Tataranni PA, 2000 Differential brain responses to satiation in obese and lean men. Diabetes 49, 838-846. [PubMed: 10905495]

Grabenhorst F, Rolls ET, 2008 Selective attention to affective value alters how the brain processes taste stimuli. Eur J Neurosci 27, 723-729. [PubMed: 18279324]

Grabenhorst F, Rolls ET, Bilderbeck A, 2008 How cognition modulates affective responses to taste and flavor: top-down influences on the orbitofrontal and pregenual cingulate cortices. Cereb Cortex 18 , 1549-1559. [PubMed: 18056086]

Grabenhorst F, Rolls ET, Parris BA, d'Souza AA, 2010 How the brain represents the reward value of fat in the mouth. Cereb Cortex 20, 1082-1091. [PubMed: 19684248]

Hassani OK, Cromwell HC, Schultz W, 2001 Influence of expectation of different rewards on behavior-related neuronal activity in the striatum. J Neurophysiol 85, 2477-2489. [PubMed: 11387394]

Haase L, Cerf-Ducastel B, Murphy C, 2009 Cortical activation in response to pure taste stimuli during the physiological states of hunger and satiety. Neuroimage 44, 1008-1021. [PubMed: 19007893]

Hosokawa T, Kato K, Inoue M, Mikami A, 2007 Neurons in the macaque orbitofrontal cortex code relative preference of both rewarding and aversive outcomes. Neurosci Res 57, 434-445. [PubMed: 17239463]

Huang CS, Hiraba H, Murray GM, Sessle BJ, 1989 Topographical distribution and functional properties of cortically induced rhythmical jaw movements in the monkey (Macaca fascicularis). $\mathrm{J}$ Neurophysiol 61, 635-650. [PubMed: 2709104]

Husain M, Roiser JP, 2018 Neuroscience of apathy and anhedonia: a transdiagnostic approach. Nat Rev Neurosci 19, 470-484. [PubMed: 29946157]

Ifuku H, Nakamura T, Hirata S, Ogawa H, 2006 Neuronal activities in the reward phase in primary and higher-order gustatory cortices of monkeys. Neurosci Res 55, 54-64. [PubMed: 16529833]

Ito S, Ogawa H, 1994 Neural activities in the fronto-opercular cortex of macaque monkeys during tasting and mastication. Jpn J Physiol 44, 141-156. [PubMed: 7967217] 
Kable JW, Glimcher PW, 2007 The neural correlates of subjective value during intertemporal choice. Nat Neurosci 10, 1625-1633. [PubMed: 17982449]

Kadohisa M, Verhagen JV, Rolls ET, 2005 The primate amygdala: Neuronal representations of the viscosity, fat texture, temperature, grittiness and taste of foods. Neuroscience 132, 33-48. [PubMed: 15780464]

Kaskan PM, Costa VD, Eaton HP, Zemskova JA, Mitz AR, Leopold DA, Ungerleider LG, Murray EA, 2017 Learned Value Shapes Responses to Objects in Frontal and Ventral Stream Networks in Macaque Monkeys. Cereb Cortex 27, 2739-2757. [PubMed: 27166166]

Kennerley SW, Wallis JD, 2009 Evaluating choices by single neurons in the frontal lobe: outcome value encoded across multiple decision variables. Eur J Neurosci 29, 2061-2073. [PubMed: 19453638]

Kringelbach ML, O'Doherty J, Rolls ET, Andrews C, 2003 Activation of the human orbitofrontal cortex to a liquid food stimulus is correlated with its subjective pleasantness. Cereb Cortex 13, 1064-1071. [PubMed: 12967923]

Kusama T, Fujioka M, Miyakawa Y, Fujii M, 1985 Connections of the fronto-parietal operculum and the postcentral gyrus with the posterior ventral thalamic nucleus, especially its medial nucleus, in monkeys. J Hirnforsch 26, 317-331. [PubMed: 2411787]

Lara AH, Kennerley SW, Wallis JD, 2009 Encoding of gustatory working memory by orbitofrontal neurons. J Neurosci 29, 765-774. [PubMed: 19158302]

Leathers ML, Olson CR, 2017 In monkeys making value-based decisions, amygdala neurons are sensitive to cue value as distinct from cue salience. J Neurophysiology 117, 1499-1511.

Málková L, Gaffan D, Murray EA, 1997 Excitotoxic lesions of the amygdala fail to produce impairment in visual learning for auditory secondary reinforcement but interfere with reinforcer devaluation effects in rhesus monkeys. J Neurosci 17, 6011-6020. [PubMed: 9221797]

Mitz AR, 2005 A liquid-delivery device that provides precise reward control for neurophysiological and behavioral experiments. J Neurosci Meth 148, 19-25.

McCabe C, Rolls ET, 2007 Umami: a delicious flavor formed by convergence of taste and olfactory pathways in the human brain. Eur J Neurosci 25, 1855-1864. [PubMed: 17432971]

Mogami T, Tanaka K, 2006 Reward association affects neuronal responses to visual stimuli in macaque TE and perirhinal cortices. J Neurosci 26, 6761-6770. [PubMed: 16793883]

Morrison SE, Salzman CD, 2009 The convergence of information about rewarding and aversive stimuli in single neurons. J Neurosci 29, 11471-11483. [PubMed: 19759296]

Murray EA, Rudebeck PH, 2018 Specializations for reward-guided decision-making in the primate ventral prefrontal cortex. Nat Rev Neurosci 19, 404-417. [PubMed: 29795133]

Murphy K, Bodurka J, Bandettini PA, 2007 How long to scan? The relationship between fMRI temporal signal to noise ratio and necessary scan duration. Neuroimage 34, 565-574. [PubMed: 17126038]

Nishijo H, Ono T, Nakamura K, Kawabata M, Yamatani K, 1986 Neuron activity in and adjacent to the dorsal amygdala of monkey during operant feeding behavior. Brain Res Bull 17, 847-854. [PubMed: 3801939]

Nishijo H, Ono T, Nishino H, 1988 Single neuron responses in amygdala of alert monkey during complex sensory stimulation with affective significance. J Neurosci 8, 3570-3583. [PubMed: 3193171]

O'Doherty J, Rolls ET, Francis S, Bowtell R, McGlone F, 2001 Representation of pleasant and aversive taste in the human brain. J Neurophysiol 85, 1315-1321. [PubMed: 11248000]

Ogawa H, 1994 Gustatory cortex of primates: anatomy and physiology. Neurosci Res 20, 1-13. [PubMed: 7984335]

Ogawa H, Ito S, Nomura T, 1989 Oral cavity representation at the frontal operculum of macaque monkeys. Neurosci Res 6, 283-298. [PubMed: 2725988]

Padoa-Schioppa C, 2011 Neurobiology of economic choice: a good-based model. Annu Rev Neurosci 34, 333-359. [PubMed: 21456961]

Padoa-Schioppa C, Assad JA, 2006 Neurons in the orbitofrontal cortex encode economic value. Nature 441, 223-226. [PubMed: 16633341] 
Paton JJ, Belova MA, Morrison SE, Salzman CD, 2006 The primate amygdala represents the positive and negative value of visual stimuli during learning. Nature 439, 865-870. [PubMed: 16482160]

Pritchard TC, Edwards EM, Smith CA, Hilgert KG, Gavlick AM, Maryniak TD, Schwartz GJ, Scott TR, 2005 Gustatory neural responses in the medial orbitofrontal cortex of the old world monkey. J Neurosci 25, 6047-6056. [PubMed: 15987934]

Pritchard TC, Hamilton RB, Morse JR, Norgren R, 1986 Projections of thalamic gustatory and lingual areas in the monkey, Macaca fascicularis. J Comp Neurol 244, 213-228. [PubMed: 3950095]

Pritchard TC, Nedderman EN, Edwards EM, Petticoffer AC, Schwartz GJ, Scott TR, 2008 Satietyresponsive neurons in the medial orbitofrontal cortex of the macaque. Behav Neurosci 122, 174182. [PubMed: 18298260]

Roberts AC, Reekie Y, Braesicke K, 2007 Synergistic and regulatory effects of orbitofrontal cortex on amygdala-dependent appetitive behavior. Ann N Y Acad Sci 1121, 297-319. [PubMed: 17698997]

Roesch MR, Olson CR, 2004 Neuronal activity related to reward value and motivation in primate frontal cortex. Science 304, 307-310. [PubMed: 15073380]

Rolls ET, Kellerhals MB, Nichols TE, 2015 Age differences in the brain mechanisms of good taste. Neuroimage 113, 298-309. [PubMed: 25842291]

Rolls ET, Scott TR, Sienkiewicz ZJ, Yaxley S,1988 The responsiveness of neurones in the frontal opercular gustatory cortex of the macaque monkey is independent of hunger. J Physiol 397, 1-12. [PubMed: 3411507]

Rolls ET, Sienkiewicz ZJ, Yaxley S, 1989 Hunger Modulates the Responses to Gustatory Stimuli of Single Neurons in the Caudolateral Orbitofrontal Cortex of the Macaque Monkey. Eur J Neurosci 1, 53-60. [PubMed: 12106174]

Rolls ET, Yaxley S, Sienkiewicz ZJ, 1990 Gustatory responses of single neurons in the caudolateral orbitofrontal cortex of the macaque monkey. J Neurophysiol 64, 1055-1066. [PubMed: 2258734]

Rudebeck PH, Mitz AR, Chacko RV, Murray EA, 2013 Effects of amygdala lesions on reward-value coding in orbital and medial prefrontal cortex. Neuron 80, 1519-1531. [PubMed: 24360550]

Rudebeck PH, Murray EA, 2014 The orbitofrontal oracle: cortical mechanisms for the prediction and evaluation of specific behavioral outcomes. Neuron, 84: 1143-1156. [PubMed: 25521376]

Rudenga KJ, Small DM, 2013 Ventromedial prefrontal cortex response to concentrated sucrose reflects liking rather than sweet quality coding. Chem Senses 38, 585-594. [PubMed: 23828907]

Rushworth MF, Noonan MP, Boorman ED, Walton ME, Behrens TE, 2011 Frontal cortex and rewardguided learning and decision-making. Neuron 70, 1054-1069. [PubMed: 21689594]

Saleem KS, and Logothetis NK, 2012 A combined MRI and histology atlas of the rhesus monkey brain in stereotaxic coordinates, 2nd Edition. Associated Press.

Schultz W, Apicella P, Scarnati E, Ljungberg T, 1992 Neuronal activity in monkey ventral striatum related to the expectation of reward. J Neurosci 12, 4595-4610. [PubMed: 1464759]

Scott TR, Mark GP, 1986 Feeding and taste. Prog Neurobiol 27, 293-317. [PubMed: 3024228]

Scott TR, Plata-Salaman CR, Smith VL, Giza BK, 1991 Gustatory neural coding in the monkey cortex: stimulus intensity. J Neurophysiol 65, 76-86. [PubMed: 1999734]

Scott TR, Yaxley S, Sienkiewicz ZJ Rolls, 1986 Gustatory responses in the frontal opercular cortex of the alert cynomolgus monkey. J Neurophysiol 56, 876-890. [PubMed: 3783223]

Simmons WK, Reddish M, Bellgowan PS, Martin A, 2010 The selectivity and functional connectivity of the anterior temporal lobes. Cereb Cortex 20, 813-825. [PubMed: 19620621]

Small DM, Zatorre RJ, Dagher A, Evans AC, Jones-Gotman M, 2001 Changes in brain activity related to eating chocolate: from pleasure to aversion. Brain 124, 1720-1733. [PubMed: 11522575]

Small DM, Jones-Gotman M, Dagher A, 2003 Feeding-induced dopamine release in dorsal striatum correlates with meal pleasantness ratings in healthy human volunteers. Neuroimage 19, 17091715. [PubMed: 12948725]

Smeets PA, de Graaf C, Stafleu A, van Osch MJ, Nievelstein RA, van der Grond J, 2006 Effect of satiety on brain activation during chocolate tasting in men and women. Am J Clin Nutr 83, 1297 1305. [PubMed: 16762940] 
Strait CE, Sleezer BJ, Blanchard TC, Azab H, Castagno MD, Hayden BY, 2016 Neuronal selectivity for spatial positions of offers and choices in five reward regions. J Neurophysiol 115, 1098-1111. [PubMed: 26631146]

Suzuki S, Cross L, O’Doherty JP, 2017 Elucidating the underlying components of food valuation in the human orbitofrontal cortex. Nat Neurosci 20, 1780-1786. [PubMed: 29184201]

Thomas JM, Higgs S, Dourish CT, Hansen PC, Harmer CJ, McCabe C, 2015 Satiation attenuates BOLD activity in brain regions involved in reward and increases activity in dorsolateral prefrontal cortex: an fMRI study in healthy volunteers. Am J Clin Nutr 101, 697-704. [PubMed: 25833968]

Tremblay L, Schultz W, 1999 Relative reward preference in primate orbitofrontal cortex. Nature 398, 704-708. [PubMed: 10227292]

van Rijn I, de Graaf C, Smeets PA, 2015 Tasting calories differentially affects brain activation during hunger and satiety. Behav Brain Res 279, 139-147. [PubMed: 25449847]

Xiang QS Ye, F.Q. 2007 Correction for geometric distortion and N/2 ghosting in EPI by phase labeling for additional coordinate encoding (PLACE). Magn Reson Med 57, 731-741. [PubMed: 17390358]

Yan J, Scott TR, 1996 The effect of satiety on responses of gustatory neurons in the amygdala of alert cynomolgus macaques. Brain Res 740, 193-200. [PubMed: 8973814]

Yaxley S, Rolls ET, Sienkiewicz ZJ, 1988 The responsiveness of neurons in the insular gustatory cortex of the macaque monkey is independent of hunger. Physiol Behav 42, 223-229. [PubMed: 3406148]

Yaxley S, Rolls ET, Sienkiewicz ZJ, 1990 Gustatory responses of single neurons in the insula of the macaque monkey. J Neurophysiol 63, 689-700. [PubMed: 2341869]

Zald DH, Lee JT, Fluegel KW, Pardo JV, 1998 Aversive gustatory stimulation activates limbic circuits in humans. Brain 121 (Pt 6), 1143-1154. [PubMed: 9648549] 


\section{Highlights}

- $\quad$ Taste responsive areas in the macaque include primary gustatory cortex area $\mathrm{G}$, an adjacent portion of the anterior insular cortex, and area 120 .

- Neural representations in the ventral striatum, ventral pallidum, and amygdala signal taste preference and/or subjective value.

- Activations in area G, the adjacent portion of the anterior insular cortex, and area $12 \mathrm{o}$ in the left hemisphere are positively related to levels of hydration, which may reflect influences of internal state on taste processing.

- Activations in medial prefrontal cortex area $32 / 10 \mathrm{~m}$ are negatively related to levels of hydration. Thus, activation in area $32 / 10 \mathrm{~m}$, a region implicated in value-based decision making, is modulated by changes in internal state. 

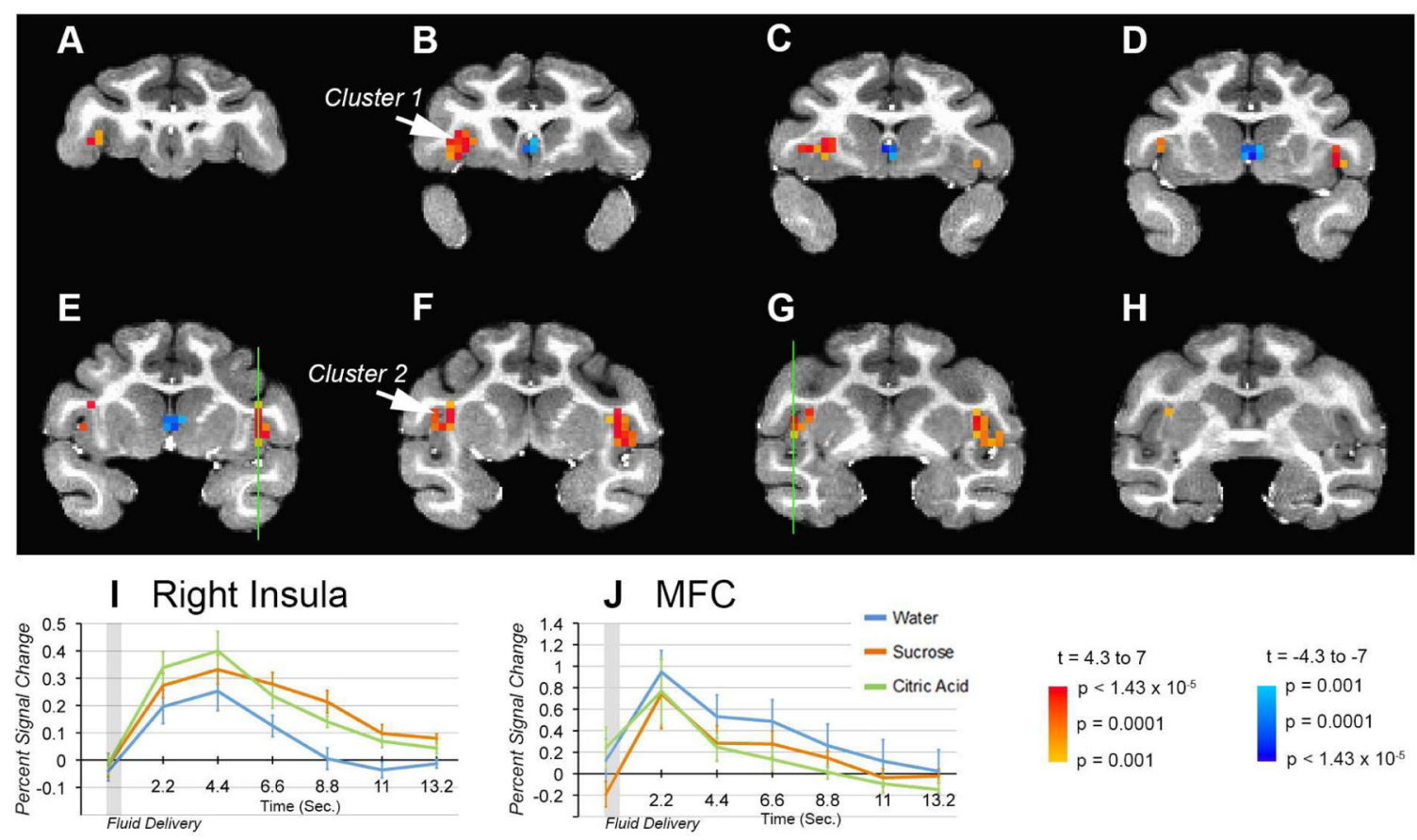

$\mathrm{t}=-4.3$ to -7

$p=0.001$

$p=0.0001$

Fluid Delivery
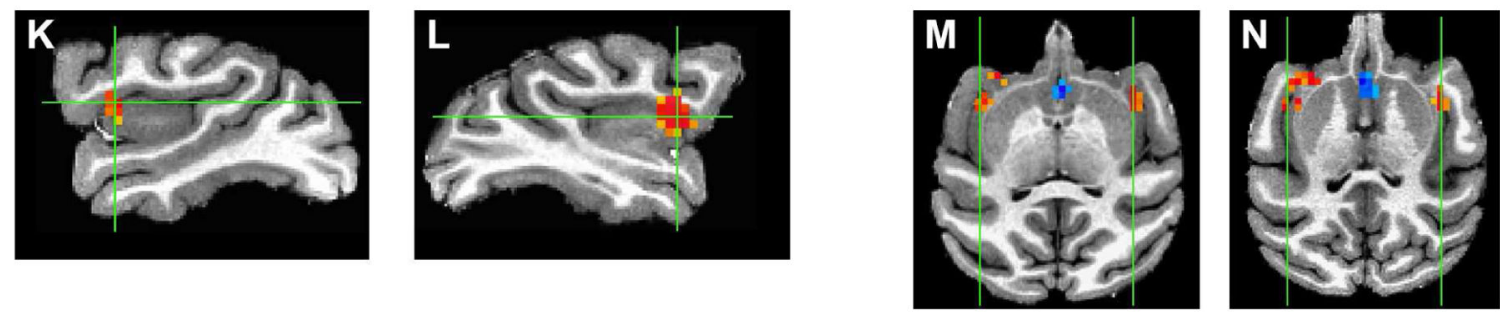

Figure 1. Taste responsive areas.

A-H. Areas exhibiting greater BOLD responses to sucrose and citric acid relative to water included area $12 \mathrm{o}$, area $\mathrm{G}$, and an adjacent portion of the anterior insula. t-maps range from $4.3(p=0.001)$ to $7.0\left(p<1.43 \times 10^{-5}\right)$ and above (red color scale), and $-4.3(p=0.001)$ to $-7.0\left(p<1.43 \times 10^{-5}\right)$ and below (blue color scale); corrected FWE $=0.01$. t-maps are masked with licking prior to fluid delivery. Sections A-H are taken at $1.5 \mathrm{~mm}$ intervals. Two distinct clusters were identified in the left insula, indicated as "Cluster 1" in panels A-D, and "Cluster 2" in panels E-H. The center of Cluster 1 is $\sim 8 \mathrm{~mm}$ rostral to the anterior commissure, and is about $13 \mathrm{~mm}$ from the midline. The center of Cluster 2 is $\sim 2 \mathrm{~mm}$ rostral to the anterior commissure, and is about $16 \mathrm{~mm}$ from the midline. The cluster in the insula in the right hemisphere spans $7.5 \mathrm{~mm}$ (Sections $\mathrm{C}-\mathrm{G}$ ) and is centered $\sim 4 \mathrm{~mm}$ rostral to the anterior commissure; it is about $19 \mathrm{~mm}$ from the midline. Note the anterior commissure in Section $\mathrm{H}$ for reference. Area 25 exhibited greater BOLD responses to water relative to sucrose and citric acid (blue voxels). I. Time course for a cluster in the right insula and operculum, including area $\mathrm{G}$, shows greater BOLD responses to sucrose and citric acid than to water. J. Time course for a cluster in MFC area 25, encroaching on the ventral striatum, shows greater BOLD responses for water than sucrose or citric acid. Time-course plots show average percent signal change for all voxels in the cluster. Error bars in $\mathbf{I}$ and $\mathbf{J}$ show +/SEM across sessions. 


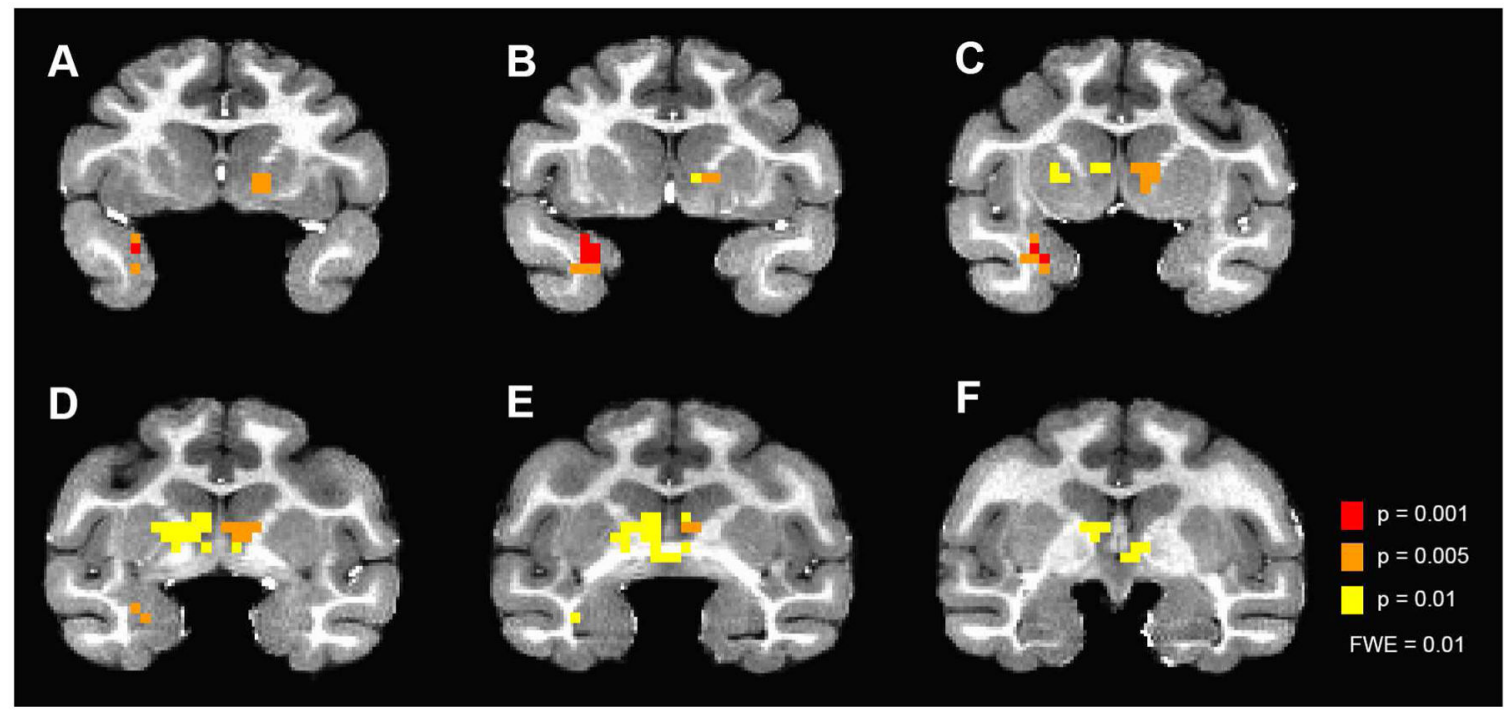

Figure 2. Ventral striatum, ventral pallidum, and amygdala encode preferred taste. t-maps at three different thresholds are shown for the sucrose vs. citric acid contrast, revealing greater BOLD responses in the ventral striatum, ventral pallidum, and left anterior amygdala for sucrose relative to citric acid. t-maps thresholded at $p=0.01, p=0.005$ and $p$ $=0.001$, each corrected at FWE 0.01. t-maps are masked with licking prior to fluid delivery. 
A Choice Preferences
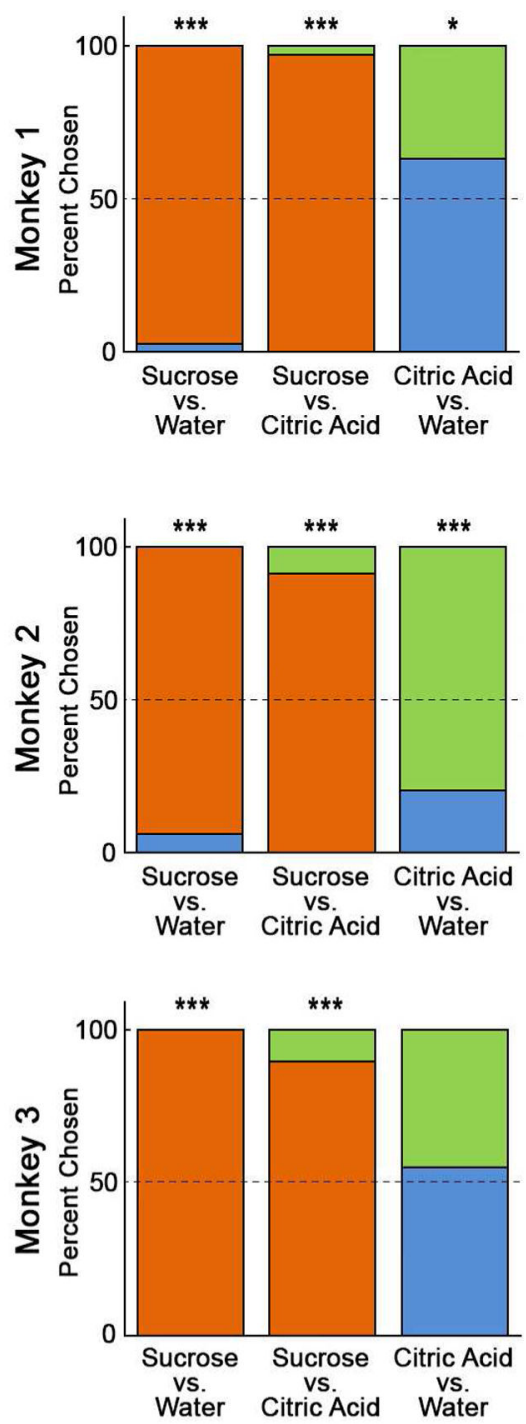
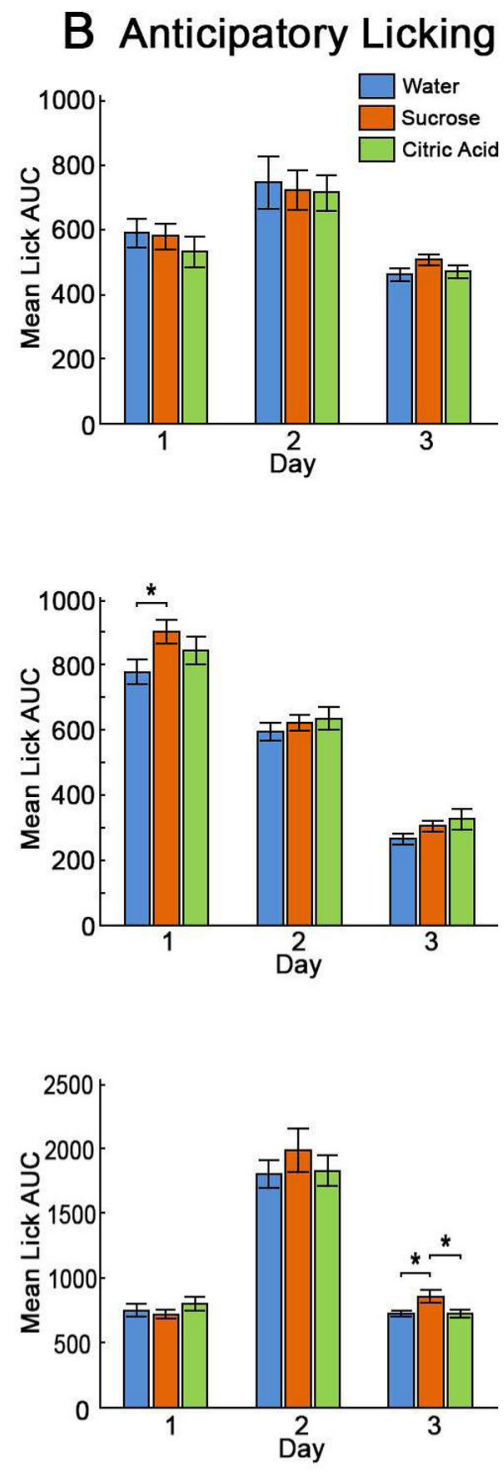

Figure 3. Choice preferences and anticipatory licking.

A. Monkeys' choice preferences from the Choice and View task for all completed choice trials. Each bar represents the percent chosen across three days for each possible pairing of fluid types: sucrose vs. water, sucrose vs. citric acid, and citric acid vs. water (see legend in B). For each bar, the fraction of color segment indicates the percent chosen. All three monkeys chose cues signaling delivery of sucrose over those signaling water (all $p$ s < $0.001)$. All three monkeys also chose cues signaling delivery of sucrose over those signaling delivery of citric acid (all $p \mathrm{~s}<0.001)$. Monkey 1 preferred water over citric acid $(p=0.015)$ and Monkey 2 preferred citric acid to water $\left(p=3.02 \times 10^{-6}\right)$, while Monkey 3 showed no preference $(p=0.457)$. B. Monkeys' anticipatory licking from the Choice and View task for all completed View trials. Licking was evaluated between cue onset $(\mathrm{t}=0)$ and the earliest fluid delivery time. Bars depict the mean (+/- 1 SEM) lick area under the curve (AUC) for each fluid type, for each day. Monkey 1 exhibited no significantly different licking cues 
associated with delivery of sucrose, citric acid, or water. Monkey 2 showed significantly greater licking in anticipation of sucrose over water on day one $(p=0.026)$. Monkey 3 licked more in anticipation of sucrose than water $(\mathrm{p}=0.024)$ or citric acid $(\mathrm{p}=0.018)$ on day three. Asterisks denote significance as follows: $p<0.001 \mathrm{sig}=* * *, p<0.01, \mathrm{sig}=* * ; p$ $<0.05, \operatorname{sig}=*$. The legend shown in panel B applies to the entire figure. 


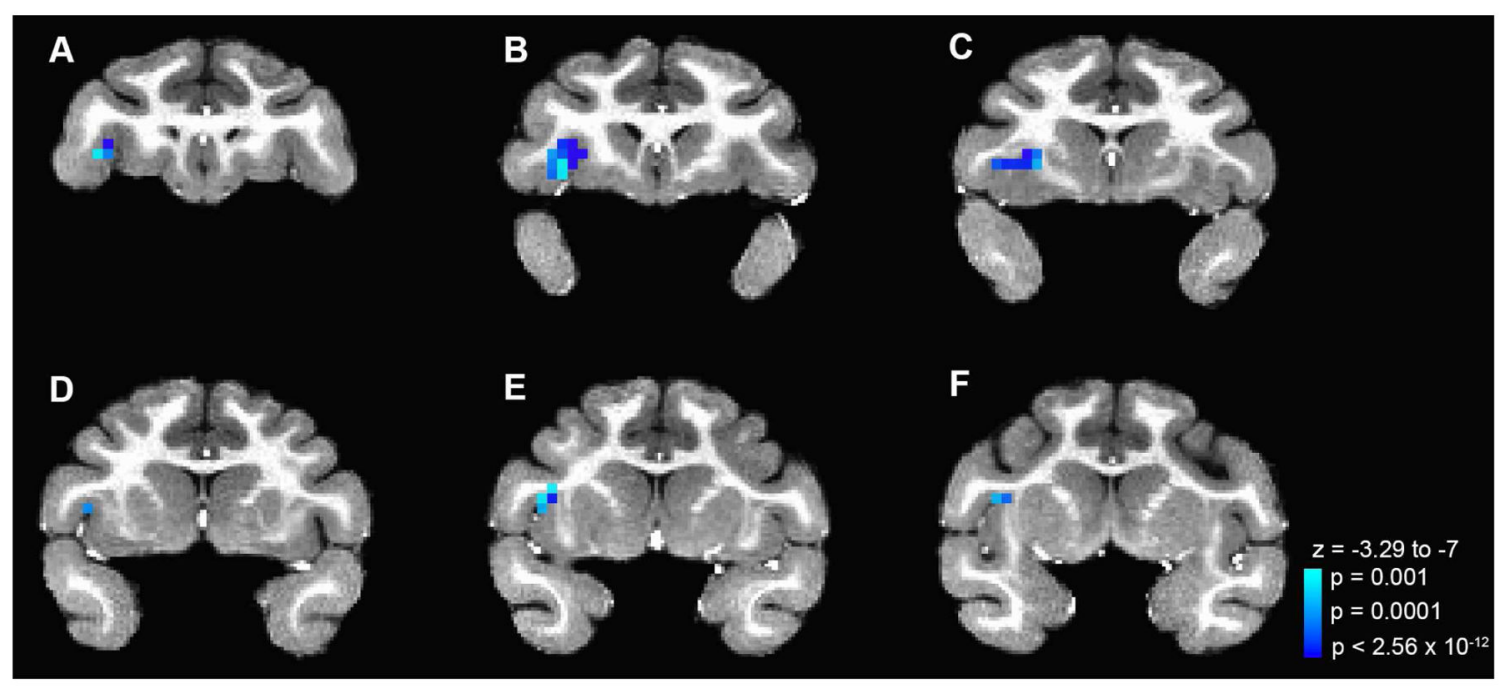

G Left $120+$ Ant. Insula

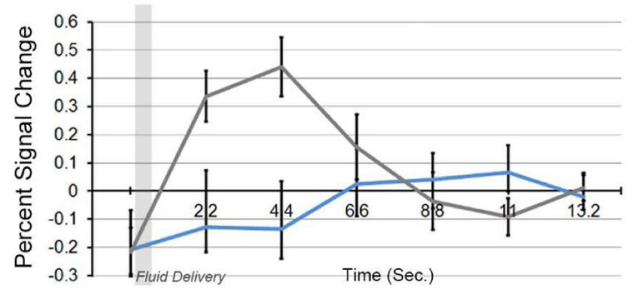

H Right Insula

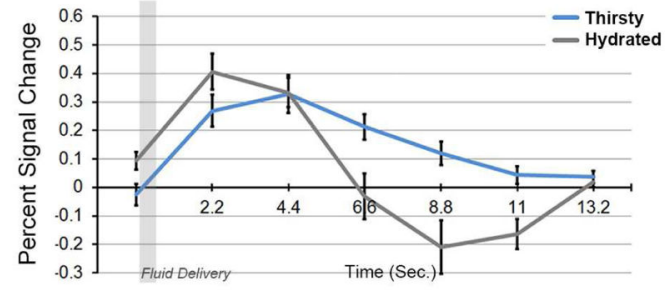

Figure 4. Taste responsive areas affected by hydration.

A-F. Of identified taste responsive areas, only area 120 and area $\mathrm{G}$ in the left hemisphere show responses modulated by hydration level. These regions exhibited greater BOLD responses following full hydration (pre minus post hydration contrast maps shown). There was no interaction between fluid type and hydration (not shown). z-maps range from -3.29 $(p=0.001)$ to $-7.0\left(p<\times 2.56 \times 10^{-12}\right)$; corrected FWE $=0.01$. Sections A-F are taken at $1.5 \mathrm{~mm}$ intervals. G-H. Mean BOLD time courses for all fluid types before and after monkeys were given ad libitum water. G. Time course for a cluster in area G, area 12o and anterior insula shows BOLD responses significantly modulated by the hydration manipulation (hydrated $>$ thirsty). H. Time course for a cluster of taste-responsive voxels in the right hemisphere does not show significant effects of hydration. Error bars in $\mathbf{G}$ and $\mathbf{H}$ show +/- SEM across sessions. Compare and contrast with Figure 1. 


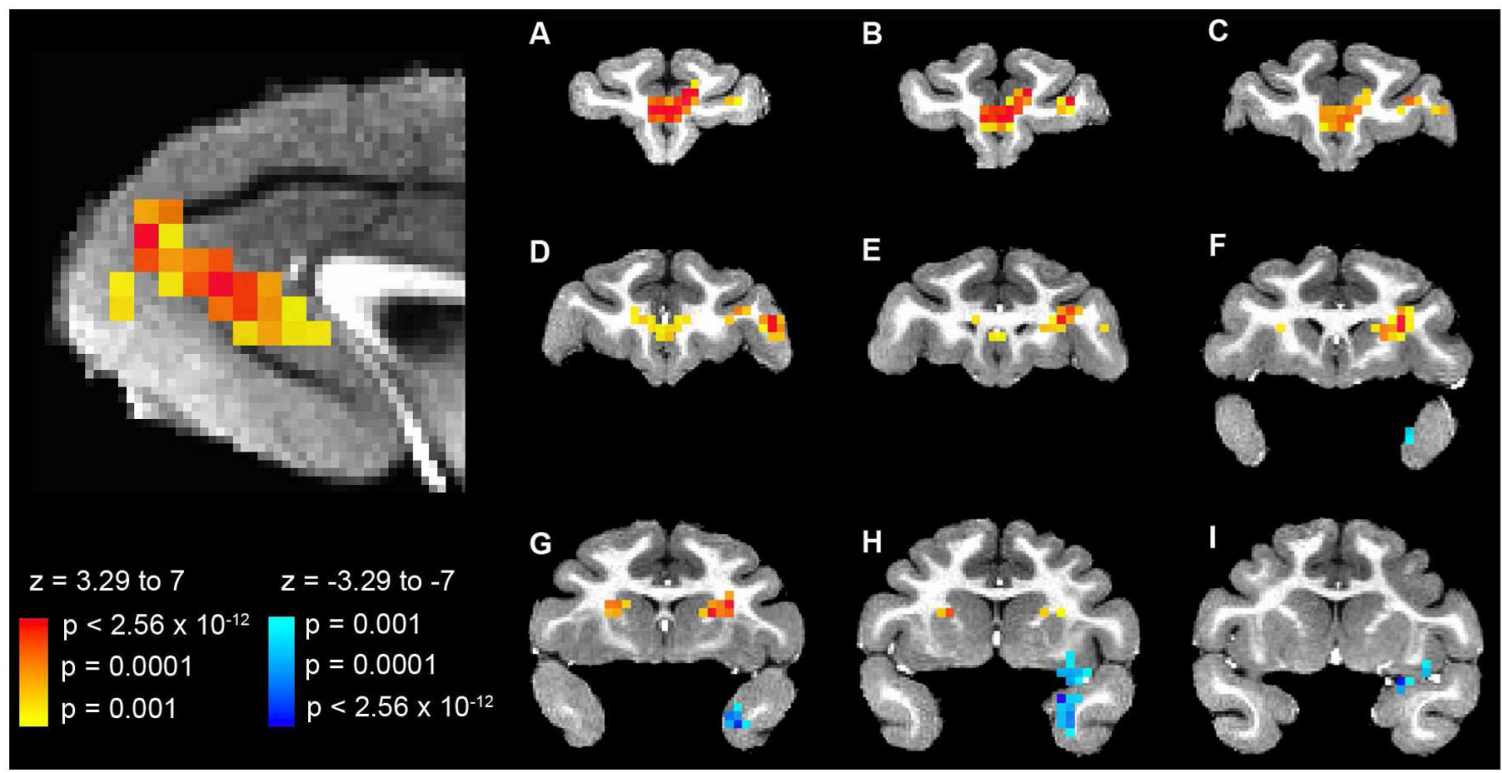

Figure 5. Brain areas affected by hydration: regions outside taste-responsive areas.

Additional areas exhibiting BOLD responses modulated by changes in hydration level included area 32/10m, a small region of cortex near the rostral extent of the ventral bank of the cingulate sulcus, area $46 \mathrm{v}$, anterodorsal striatum, and $12 \mathrm{r}$ in the right hemisphere. These regions exhibited greater BOLD responses prior to full hydration (thirsty $>$ hydrated). $\mathrm{z}$ maps range from $3.29(p=0.001)$ to $7.0\left(p<\times 2.56 \times 10^{-12}\right)$ and above; corrected FWE $=$ 0.01 . 